\title{
Resveratrol alleviates inflammatory injury and enhances the apoptosis of fibroblast-like synoviocytes via mitochondrial dysfunction and ER stress in rats with adjuvant arthritis
}

\author{
JINSEN LU ${ }^{1}$, YONGSHUN ZHENG ${ }^{2}$, JIAZHAO YANG $^{1}$, JUNQIANG ZHANG $^{2}$, \\ WEI CAO ${ }^{2}$, XIAOYU CHEN $^{2}$ and SHIYUAN FANG ${ }^{1}$ \\ ${ }^{1}$ Department of Orthopedics, Anhui Provincial Hospital, Anhui Medical University, Hefei, Anhui 230001; \\ ${ }^{2}$ Department of Histology and Embryology, Anhui Medical University, Hefei, Anhui 230032, P.R. China
}

Received October 6, 2018; Accepted March 28, 2019

DOI: $10.3892 / \mathrm{mmr} .2019 .10273$

\begin{abstract}
Resveratrol, a bioactive compound predominantly found in grapes and red wine, provides a wide range of properties that are beneficial for health, including anticancer and anti-inflammatory activities. Previously published studies have addressed the potential therapeutic effects of resveratrol on rheumatoid arthritis (RA); however, the subcellular mechanism remains to be fully elucidated. In the present study, the therapeutic effects of resveratrol on adjuvant arthritis (AA) in Sprague-Dawley rats were investigated, and the mechanisms of resveratrol-induced apoptosis in fibroblast-like synoviocytes (FLSs) were further examined. Based on the findings, resveratrol treatment over a 12-day period led to a reduction in paw swelling and arthritis scores at the macroscopic level, and an attenuation of inflammatory cell infiltration and synovial hyperplasia, upon a histopathological examination of the AA rats. Furthermore, the administration of resveratrol triggered decreases in the expression of interleukin (IL)-1, IL-6, IL-8 and tumor necrosis factor- $\alpha$ (TNF- $\alpha$ ) and an increase in the expression of IL-10, alleviating inflammatory injury in AA rats in a dose-dependent manner. In addition, resveratrol was revealed to induce the apoptosis of FLSs when administered with $5 \mu \mathrm{M} \mathrm{H}_{2} \mathrm{O}_{2}$ as determined by elevated levels of $\mathrm{Bax}$, caspase-3, caspase-12 and C/EBP-homologous protein, and the downregulation of $\mathrm{B}$-cell lymphoma 2 (Bcl-2), suggesting that
\end{abstract}

Correspondence to: Professor Shiyuan Fang, Department of Orthopedics, Anhui Provincial Hospital, Anhui Medical University, 17 Lujiang Road, Hefei, Anhui 230001, P.R. China

E-mail: fangshiyuan2008@126.com

Professor Xiaoyu Chen, Department of Histology and Embryology, Anhui Medical University, 81 Meishan Road, Hefei, Anhui 230032, P.R. China

E-mail: chenxy@ahmu.edu.cn

Key words: resveratrol, apoptosis, mitochondrial dysfunction, endoplasmic reticulum stress, fibroblast synovialcytes, adjuvant arthritis resveratrol is able to induce apoptosis in FLSs via the mitochondrial pathway and endoplasmic reticulum (ER) stress in a milieu containing $5 \mu \mathrm{M} \mathrm{H}_{2} \mathrm{O}_{2}$. Furthermore, JC-1 was used as a fluorescent probe to detect the mitochondrial membrane potential $(\Delta \psi \mathrm{m})$, and resveratrol was shown to reduce the $\Delta \psi \mathrm{m}$ in FLSs in the presence of $5 \mu \mathrm{M} \mathrm{H}_{2} \mathrm{O}_{2}$. However, resveratrol was not able to trigger intracellular calcium overload, although it did suppress ATP- and thapsigargin-induced calcium release from the ER. In conclusion, the present study revealed that resveratrol was able to alleviate inflammatory injury in AA rats, triggering the apoptosis of FLSs via the mitochondrial pathway and ER stress. These results provide a theoretical basis for future treatments using resveratrol for RA.

\section{Introduction}

Rheumatoid arthritis (RA), an immune-mediated inflammatory disease of connective tissue, chronically accelerates the erosion of cartilage and subchondral bone, characterized by symmetrical inflammation in the joints of the hands and feet $(1,2)$. Hyperplasia of fibroblast-like synoviocytes (FLSs) in pannus, the aggressive front of synovial tissue, accompanied by the mass infiltration of macrophages and lymphocytes, are the typical features of the pathogenesis in RA (3). Inflammatory and anti-inflammatory mediators exert significant effects in the pathogenesis of RA (4-6). Previous studies have demonstrated that the enhanced proliferation and attenuated apoptosis of FLSs contribute to the invasion and destruction of connective tissue by pannus in joints $(7,8)$. Through the persistent efforts of investigators, a number of natural and synthetic anti-inflammatory and anticancer drugs have been found to suppress the abnormal proliferation of FLSs in patients with RA, or in adjuvant arthritis (AA) model rats $(9-12)$.

Resveratrol, a bioactive compound predominantly found in grapes and red wine, provides a wide range of properties that are beneficial for health, including anticancer, anti-inflammatory, antioxidant and cardiovascular protective activities (13). Furthermore, it has been suggested that resveratrol may not only suppress proliferation, but may also induce apoptosis in various types of cancer, including 
myeloid, breast, lung, liver, pancreatic, prostate, colon and skin cancer (14-16). The mechanism by which resveratrol exerts its pro-apoptotic activities involves a wide range of signaling pathways, including the mitochondrial, caspase 8- or 9-dependent, receptor-dependent pathways and cell cycle arrest. Resveratrol-induced apoptosis has also been demonstrated to be associated with the B-cell lymphoma 2 (Bcl-2)-associated $\mathrm{X}$ protein $(\mathrm{Bax}) / \mathrm{Bcl}-2$ ratio, driven by changes in the transcriptional activity of P53 and nuclear factor- $\kappa \mathrm{B}(15,17)$. A previous study indicated that reactive oxygen species (ROS) can function as upstream signaling molecules that exert an influence on endoplasmic reticulum (ER) stress-mediated apoptosis (18). In addition, overexpressed ROS are able to induce the dysfunction of ER foldase and chaperones, leading to an accumulation of unfolded protein, reduced protein biosynthesis and alleviation of the burden of the ER, which may afford a protective response (19). However, continuous and marked ER stress may lead to a prolonged period of marked C/EBP homologous protein (Chop) expression, steering the cells towards apoptosis $(20,21)$. In our previous study, it was demonstrated that levels of mitochondrial ROS and apoptosis were elevated following resveratrol treatment in FLSs (22). Based on the close biological association of mitochondria with the ER, the pro-apoptotic effects of resveratrol may correlate with mitochondrial dysfunction and ER stress. Furthermore, excessive ROS may trigger the oxidative modification of ER proteins and suppress the $\mathrm{Ca}^{2+}$-ATPase, leading to the depletion of calcium stores and cell apoptosis, as revealed in hypoxic-ischemic model rats (21). Cytokines are vital agents in the inflammatory process. Markedly pro-inflammatory cytokines, including interleukin (IL)-1, IL-6, IL- 8 and tumor necrosis factor- $\alpha$ (TNF- $\alpha)$, combined with markedly anti-inflammatory IL-10, are ideal markers to assess the properties of resveratrol and its influence on immune responses (4-6).

Considering the experimental evidence amassed to date, resveratrol may be considered as a potential alleviator of the symptoms of RA for patients, although its specific effects on FLSs and the underlying mechanism remain to be elucidated. The present study aimed to further investigate the pro-apoptotic mechanism of resveratrol on FLSs with respect to mitochondrial and ER dysfunction.

\section{Materials and methods}

Animal groupings and model evaluation. A total of 60

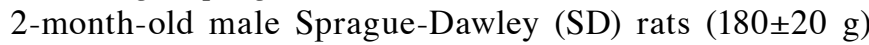
were obtained from the Laboratory Animal Center of Anhui Medical University (Hefei, China). The present study was approved by the Medical Ethics Committee of the Academic Committee at Anhui Medical University. The animals were acclimatized in a room at constant temperature $\left(22-23^{\circ} \mathrm{C}\right)$ under a 12-h light-dark cycle (light on at 7:00 AM), and the relative humidity was controlled within the range $50-70 \%$. The rats were provided with access to standard mice chow and water ad libitum. Aliquots of $150 \mu$ l Freund's complete adjuvant (FCA; Sigma-Aldrich; Merck KGaA, Darmstadt, Germany) were injected into the left hind toe of the male SD rats once, and evident arthritis was observed 20 days later. Control rats were injected with $150 \mu$ l physiological saline in the identical region of the limb. Subsequently, the AA model rats were randomly divided into five groups that were respectively treated with $0,5,15$ or $45 \mathrm{mg} / \mathrm{kg}$ resveratrol and $200 \mathrm{mg} / \mathrm{kg} N$-acetyl-L-cysteine (NAC; Merck KGaA) for 12 days by continuous intragastric administration. The selection of the doses used in the present study were based on a study described previously (23). All animals were sacrificed on the 12th day upon completion of the above-mentioned treatments. Blood samples were centrifuged at $1,500 \mathrm{x} g$ for $20 \mathrm{~min}$ at $-4^{\circ} \mathrm{C}$ to obtain the supernatant fluid, which was stored at $-80^{\circ} \mathrm{C}$ prior to further analysis. The degree of paw swelling and the arthritis index scores were determined to evaluate the severity of AA. Following the injection of the FCA emulsion, the hind paw volume (ml) of all rats was measured using a plethysmometer at 3-day intervals. The arthritis index was classified using a four-points scale ( 0 points, red spots or mild swelling; 2 points, moderate joint swelling; 3 points, severe joint swelling; 4 points, joint rigidity, deformity or severe dysfunction), totaling 16 points for each rat. The degree of paw swelling was also used to evaluate the severity of the lesion in the affected limb. Prior to the induction of inflammation, an animal volume detector (Jinan Yanyi Biotechnology Corporation, Jinan, China) was applied to detect the affected hind paw volume of each rat (F0). From the onset of inflammation, the left hind paw volume was measured every 4 days (F1), and the degree of paw swelling of the affected paw was calculated according to F1/F0.

Cell culture and isolation. FLSs were isolated from the rats in the AA model group as described previously (24). Sterile synovial tissue samples were separated into $1-\mathrm{mm}^{3}$-sized pieces, mixed with twice the volume of $0.2 \%$ type II collagenase (Merck KGaA) containing $10 \% \mathrm{Gibco}^{\circledR}$ fetal bovine serum (FBS; Thermo Fisher Scientific, Inc., Waltham, MA, USA), and the tissues were digested for $2-2.5 \mathrm{~h}$ at $25^{\circ} \mathrm{C}$ (percussing the samples once every $30 \mathrm{~min}$ ). Finally, $0.25 \%$ trypsin was added for a further digestion step for $30 \mathrm{~min}$, and the isolated FLSs were cultured in Gibco ${ }^{\circledR}$ Dulbecco's modified Eagle medium (Thermo Fisher Scientific, Inc.) supplemented with 15\% FBS in an incubator at $37^{\circ} \mathrm{C}$ with $5 \% \mathrm{CO}_{2}$ prior to performing the following experiments.

Evaluation of inflammatory injury, apoptosis and intracellular ROS. Blood from the AA rats was obtained via removal of the eyes, and serum was isolated via centrifugation $(2,000 \mathrm{x} \mathrm{g})$ at $4^{\circ} \mathrm{C}$ for $20 \mathrm{~min}$. Serum levels of IL-1, IL-6, IL-8, IL10 and TNF- $\alpha$ were detected using ELISA kits (all Abcam, Cambridge, MA, USA) for mouse IL-1 (cat. no. ab100704), IL-6 (cat. no. ab100712), IL-8 (cat. no. ab46032), IL10 (cat. no. ab108870) and TNF- $\alpha$ (cat. no. ab208348) according to the manufacturer's protocol. Cell apoptosis was detected using a terminal deoxynucleotidyl-transferase-mediated dUTP nick end labeling (TUNEL) assay (Roche Diagnostics, Indianapolis, IN, USA) in situ cell death detection kit (cat. no. 11684817910), according to the manufacturer's protocol. Briefly, FLSs were washed 3 times with phosphate-buffered saline (PBS) and incubated with reaction buffer at $37^{\circ} \mathrm{C}$ for $30 \mathrm{~min}$ in the dark. FLS were then stained with DAPI at room temperature for $5 \mathrm{~min}$ in the dark to visualize the nuclei, following which slices were mounted (cat. no. ab64230; 
Abcam) at room temperature for $\sim 5 \mathrm{~min}$. The number of apoptotic cells, and the total numbers of cells, were counted from five random fields in each slide under a light microscope (magnification, $\mathrm{x} 200$ ). The results are presented as the ratio of the apoptotic cell number to the total cell number $(n=3$ for each group) (25).

Apoptosis-associated proteins, including Bcl-2, caspase-3, Bax, caspase-12 and Chop, were detected via western blot analysis according to a protocol described previously (23). Briefly, cells were lysed using RIPA buffer (Beyotime Institute of Technology, Shanghai, China) and centrifuged at $12,000 \mathrm{x} \mathrm{g}$ for $10 \mathrm{~min}$ at $4^{\circ} \mathrm{C}$. The protein concentration was determined using a BCA Protein Assay kit (cat. no. P0011; Beyotime Institute of Technology). The supernatants were degenerated via heating at $100^{\circ} \mathrm{C}$ for $5 \mathrm{~min}$ with $1 / 5$ volume of loading buffer (Beyotime Institute of Technology), and $30 \mu \mathrm{g}$ samples were loaded in each well, separated via 10\% SDS-PAGE and transferred to PVDF membranes (EMD Millipore, Billicera, MA, USA). Next, 5\% nonfat milk in washing buffer was used to block the PVDF membranes for $2 \mathrm{~h}$ at room temperature, which were then incubated at $4^{\circ} \mathrm{C}$ overnight with primary antibodies (all from Abcam) specific for Bax (1:1,500; cat. no. ab32503), Bcl-2 (1:2,000; cat. no. ab182858), caspase-3 (1:2,000; cat. no. ab13847), caspase-12 (1:2,500; cat. no. ab62484), Chop $(1: 3,000$; cat. no. ab179823) and $\beta$-actin $(1: 10,000$; cat. no. ab115777). On the following day, membranes were washed and incubated with horseradish peroxidase-conjugated anti-rabbit Immunoglobulin G secondary antibody $(1: 10,000$; cat. no. A0208; Beyotime Institute of Biotechnology) for $1 \mathrm{~h}$ at room temperature. Finally, protein bands were visualized using enhanced chemiluminescence reagent (Boster Biological Technology, Pleasanton, CA, USA), imaged using a Gel-Dox $\mathrm{XR}+$ imager (Bio-Rad Laboratories, Inc., Hercules, CA, USA) and quantified using Image Lab v4.0 (Bio-Rad Laboratories, Inc.).

2',7'-Dichlorodihydrofluorescein diacetate (DCFH-DA) was utilized as the fluorescent probe to detect intracellular ROS in the FLSs, also according to the manufacturer's protocol (Bioluminor Biotechnology Co., Ltd., Xiamen, Fujian, China). Briefly, $1 \times 10^{5} / \mathrm{cm}^{2}$ FLSs grown on glass coverslips were cultured with $5 \mu \mathrm{M}$ DCFH-DA at $37^{\circ} \mathrm{C}$ for $20 \mathrm{~min}$. Following staining, the slides were rinsed three times with PBS. Subsequently, the fluorescence intensity was determined using a confocal laser scanning microscope (Leica SP5-DMI6000-DIC; Leica Microsystems GmbH, Wetzlar, Germany); its in-built evaluation software (Leica LAS AF Lite 2.6.0 build 7288) was used to detect the quantitative analysis of the green fluorescence signal with an excitation wavelength of $488 \mathrm{~nm}$ and an emission wavelength of $522 \mathrm{~nm}$. The ROS level was positively correlated with fluorescent intensity.

Hematoxylin and eosin $(H \& E)$ staining. Following corresponding animal experiments, the rats were sacrificed via cervical dislocation. The knee joint was extracted and fixed in $4 \%$ paraformaldehyde at $4^{\circ} \mathrm{C}$. Subsequently, the tissues were dehydrated in ethanol and finally embedded in paraffin. Histologic cuts from the paraffin blocks (5-mm thickness) were obtained and stained with hematoxylin and eosin as previously described (22). Briefly, the sections were attached to the slide and heated at $60^{\circ} \mathrm{C}$. Next, the sections were dewaxed with xylene twice for $5 \mathrm{~min}$, rehydrated in a graded ethanol series and then distilled water for $3 \mathrm{~min}$, and then stained with hematoxylin for $5 \mathrm{~min}$ and with eosin for $20 \mathrm{sec}$ (both steps at room temperature). Sections were then dehydrated in a graded ethanol series and subsequently incubated with xylene for $5 \mathrm{~min}$. Finally, sections were sealed with neutral paraffin. The images of the stained tissue were captured via a light microscope (magnification, x100 and x400). A total of six fields were randomly selected from each group, and the fields of view were analyzed by two different observers.

Mitochondrial membrane potential $(\Delta \psi m)$ determination. According to the manufacturer's protocol, a $\Delta \psi \mathrm{m}$ assay kit with JC-1 (Thermo Fisher Scientific, Inc.) was used to detect $\Delta \psi \mathrm{m}$. In terms of the functioning of the assay, the JC-1 probe accumulates in the mitochondrial matrix to form a polymer, which results in marked red fluorescence in normal mitochondria; however, the JC-1 probe exists as a monomer in damaged mitochondria, and appears as marked green fluorescence. Following the specific experimental treatments with resveratrol and $\mathrm{H}_{2} \mathrm{O}_{2}, 1 \times 10^{5} / \mathrm{cm}^{2}$ FLSs, grown on glass coverslips, were cultured with $5 \mu \mathrm{g} / \mathrm{ml} \mathrm{JC}-1$ at $37^{\circ} \mathrm{C}$ for $20 \mathrm{~min}$. Following staining, the slides were rinsed three times with PBS. As described above, the fluorescence intensity was subsequently determined using a Leica SP5-DMI6000-DIC confocal laser scanning microscope (Leica Microsystems $\mathrm{GmbH}$ ), and its in-built evaluation software (Leica LAS AF Lite) was used to detect the quantitative analysis of the green fluorescence signal, with an excitation wavelength of $507 \mathrm{~nm}$ and an emission wavelength of $529 \mathrm{~nm}$. Measurements for $\Delta \psi \mathrm{m}$ were determined as the ratios of red-to-green fluorescence intensity.

Intracellular calcium $\left[\left(\mathrm{Ca}^{2}+\right)_{i}\right]$ measurement. The measurement of $\left[\mathrm{Ca}^{2+}\right]_{\mathrm{i}}$ was performed as previously described (26). Briefly, $1 \times 10^{5} / \mathrm{cm}^{2}$ FLSs were seeded on circular coverslips and incubated with $10 \mu \mathrm{mol} / 1$ Fluo- 8 combined with $0.02 \%$ pluronic acid $\mathrm{F}-127$ at $37^{\circ} \mathrm{C}$ for $20 \mathrm{~min} . \mathrm{Ca}^{2+}$ release was triggered via treating FLSs with $4 \mu \mathrm{mol} / 1$ thapsigargin (TG) or $100 \mu \mathrm{mol} / 1$ ATP for $5 \mathrm{~min}$ in $\mathrm{Ca}^{2+}$-free PBS containing $140 \mathrm{mmol} / \mathrm{l} \mathrm{NaCl}$, $5 \mathrm{mmol} / 1 \mathrm{KCl}, 1 \mathrm{mmol} / 1 \mathrm{MgCl}_{2}, 10 \mathrm{mmol} / 1$ glucose, $0.2 \mathrm{mmol} / 1$ EGTA and $5 \mathrm{mmol} / \mathrm{l}$ 4-(2-hydroxyethyl)-1-piperazineethanesulphonic acid (pH 7.4). Real-time fluctuations in fluorescence representing $\left[\mathrm{Ca}^{2+}\right]_{\mathrm{i}}$ (the internal concentration of $\mathrm{Ca}^{2+}$ ) were recorded using a Leica SP5-DMI6000-DIC confocal laser scanning microscope (Leica Microsystems $\mathrm{GmbH}$ ) with an excitation wavelength of $488 \mathrm{~nm}$ and a long-pass emission wavelength of $515 \mathrm{~nm}$. Variations in $\left[\mathrm{Ca}^{2+}\right]_{\mathrm{i}}$ are shown as the ratio of fluorescence relative to the intensity prior to the administration of TG or ATP (F1/F0). The fluorescence intensities were measured, based on an average of 20-30 cells for each measurement.

Statistical analysis. The results were analyzed using SPSS 19.0 statistical software (IBM Corp., Armonk, NY, USA), and data are shown as the mean \pm standard deviation of experiments performed in triplicate. All datasets were analyzed using one-way analysis of variance followed by Tukey's post hoc test to compare two groups. $\mathrm{P} \leq 0.05$ was considered to indicate a statistically significant difference. 

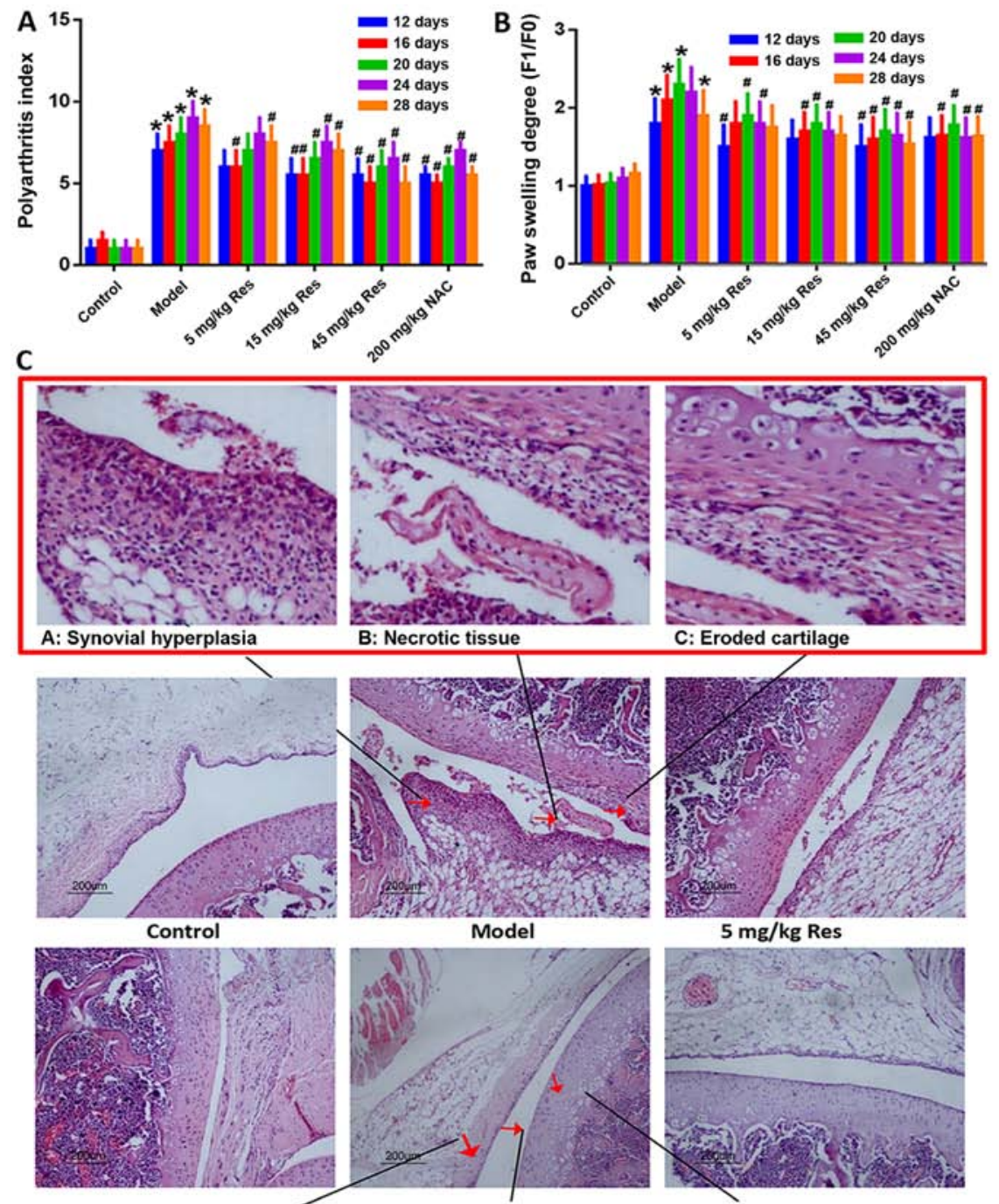

$5 \mathrm{mg} / \mathrm{kg}$ Res

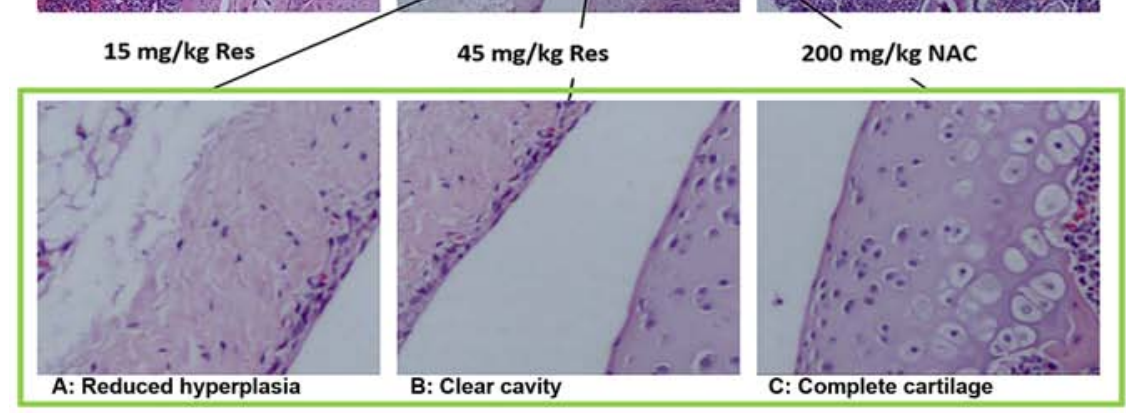

Figure 1. Representative histograms showing the (A) degree of paw swelling and (B) arthritis score in grouped Sprague-Dawley rats following intragastric administration of the respective drugs. (C) Hematoxylin and eosin staining of the knee joints in grouped adjuvant arthritis rats following intragastric administration. Specific pathological features in the model group and $45 \mathrm{mg} / \mathrm{kg}$ Res-treatment group are labeled with red arrows (magnification of expanded images, $\mathrm{x} 400)$. Data are shown as the mean \pm standard deviation. ${ }^{*} \mathrm{P}<0.05$, vs. control; ${ }^{*} \mathrm{P}<0.05$ vs. model ( $\mathrm{n}=10 /$ group). Res, resveratrol; NAC, $N$-acetyl-L-cysteine; F1/F0, fluorescence density pre-/post-agonist administration.

\section{Results}

Resveratrol attenuates the severity of arthritis induced by adjuvant in AA rats. Following the injection of FCA in SD rats, marked paw swelling was observed. In order to identify the association between the dose and pharmacological action of resveratrol, three experimental groups, treated with low $(5 \mathrm{mg} / \mathrm{kg})$, middle $(15 \mathrm{mg} / \mathrm{kg})$ and high $(45 \mathrm{mg} / \mathrm{kg})$ doses of resveratrol, were established in the present study $(23,27)$. NAC, the precursor of reduced glutathione, is an important non-enzymatic antioxidant in the cell that eliminates reactive oxygen groups, and has been demonstrated to be effective in suppressing AA and RA. Therefore, NAC was applied as a positive control in order to examine the functional role of resveratrol (28-30). Compared with the AA model group, treatment with resveratrol markedly reduced paw swelling and the arthritis score in AA rats (Fig. 1A and B). In addition, the effects of resveratrol on AA rats were further demonstrated by $\mathrm{H} \& \mathrm{E}$ staining. FCA injection triggered mass mononuclear cell infiltration, and hyperplasia of synovial tissue. Upon microscopic examination, eroded cartilage and thickened synovial tissue were clearly observed, with a large number of fragments 

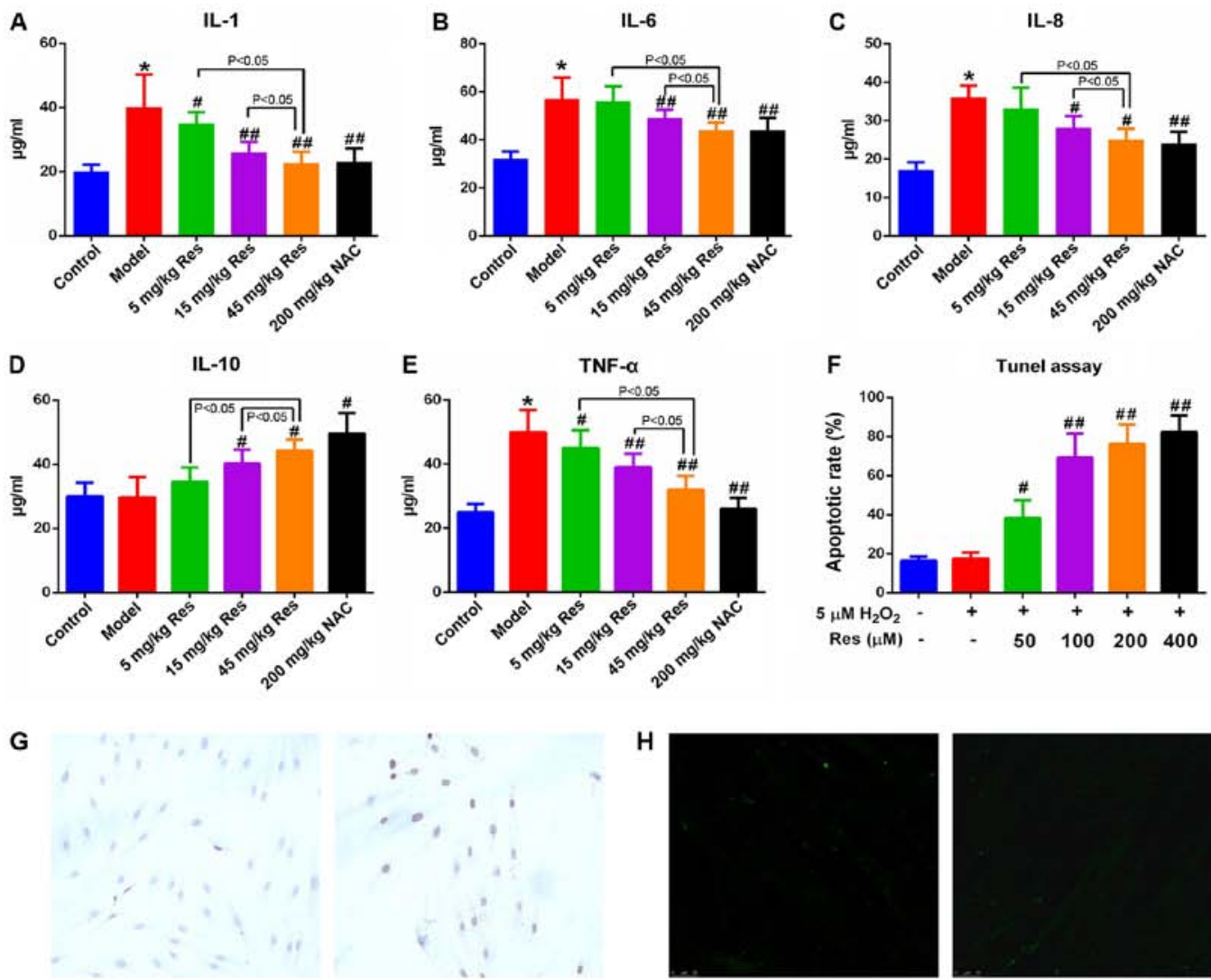

Tunel assay

G

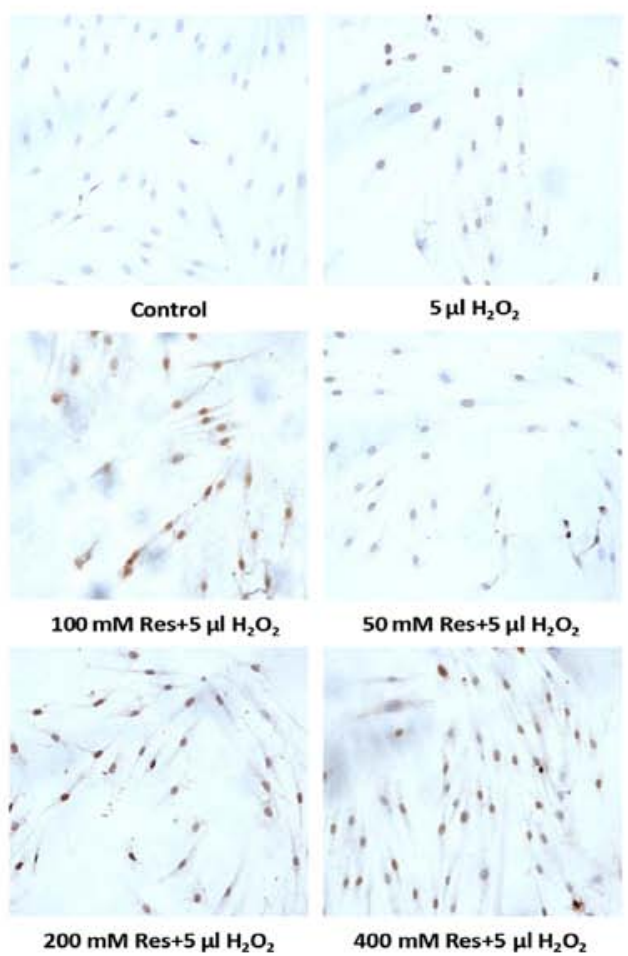

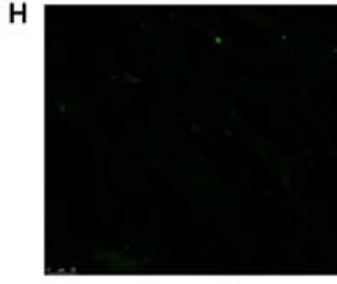

Control

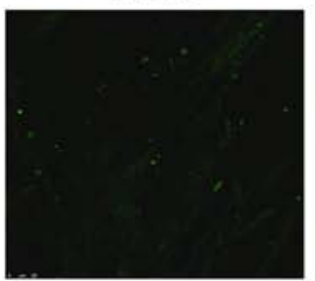

$100 \mathrm{mM}$ Res $+5 \mathrm{\mu l} \mathrm{H}_{2} \mathrm{O}_{2}$

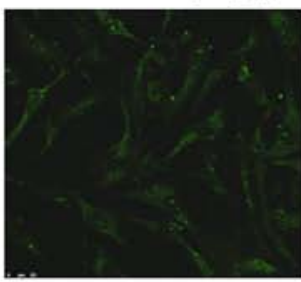

$200 \mathrm{mM}$ Res $+5 \mu \mathrm{l} \mathrm{H}_{2} \mathrm{O}_{2}$

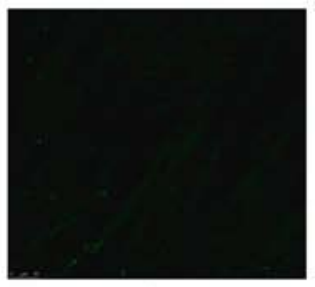

$5 \mu \mathrm{l} \mathrm{H} \mathrm{O}_{2}$

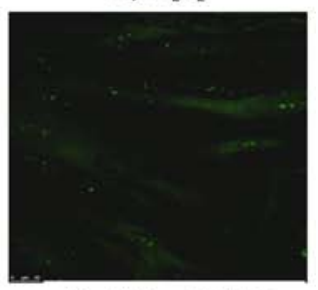

$50 \mathrm{mM}$ Res+5 $\mathrm{HI} \mathrm{H}_{2} \mathrm{O}_{2}$

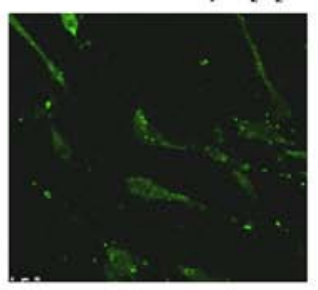

$400 \mathrm{mM}$ Res $+5 \mu \mathrm{I} \mathrm{H}_{2} \mathrm{O}_{2}$

Figure 2. Res alleviates inflammatory injury in adjuvant arthritis rats and triggers apoptosis in FLSs. Representative histograms show the serum levels of (A) IL-1, (B) IL-6, (C) IL-8, (D) IL-10 and (E) TNF- $\alpha$, from all grouped rats detected via ELISA assay. (F) Quantified data and (G) images (magnification, x400) showing the apoptotic rates of FLSs following Res and $\mathrm{H}_{2} \mathrm{O}_{2}$ treatment, detected via terminal deoxynucleotidyl-transferase-mediated dUTP nick end labeling assay. The apoptotic rate was determined by apoptotic cell number/total cell number. $(\mathrm{H})$ Representative images (magnification, $\mathrm{x} 400)$ show the intracellular reactive oxygen species levels in FLSs, detected via DCFH-DA following Res and $\mathrm{H}_{2} \mathrm{O}_{2}$ treatment. Data are shown as the mean \pm standard deviation. ${ }^{*} \mathrm{P}<0.05$, vs. control; ${ }^{\#} \mathrm{P}<0.05,{ }^{\# \#} \mathrm{P}<0.01$, vs. model ( $\mathrm{n}=10$ /group). FLSs, fibroblast-like synoviocytes; Res, resveratrol; NAC, $N$-acetyl-L-cysteine; IL, interleukin; TNF- $\alpha$, tumor necrosis factor- $\alpha$; DCFH-DA, 2',7'-dichlorodihydrofluorescein diacetate.

gathered in the synovial cavity of the AA model group. Following treatment with resveratrol, the synovial samples from rats revealed evidently attenuated inflammatory cell infiltration and synovial hyperplasia; however, all the above symptoms were relieved, or even eliminated, compared with the AA model group (Fig. 1C).

Resveratrol alleviates inflammatory injury in AA rats. The rats in the respective groups were fed for 27 days, and subsequently received intragastric administration of 5,15 or $45 \mathrm{mg} / \mathrm{kg}$ resveratrol and $200 \mathrm{mg} / \mathrm{kg}$ NAC for 12 days. Subsequently, the serum levels of IL-1, IL-6, IL-8, IL-10 and TNF- $\alpha$ were detected using ELISA assay. Administration of resveratrol led to a marginal decrease in the levels of IL-1, IL-6, IL-8 and TNF- $\alpha$, although the level of IL-10 in AA rats was increased compared with that in the non-immunized controls in a dose-dependent manner. This indicated that resveratrol was able to reduce the level of inflammatory injury in the AA rats 


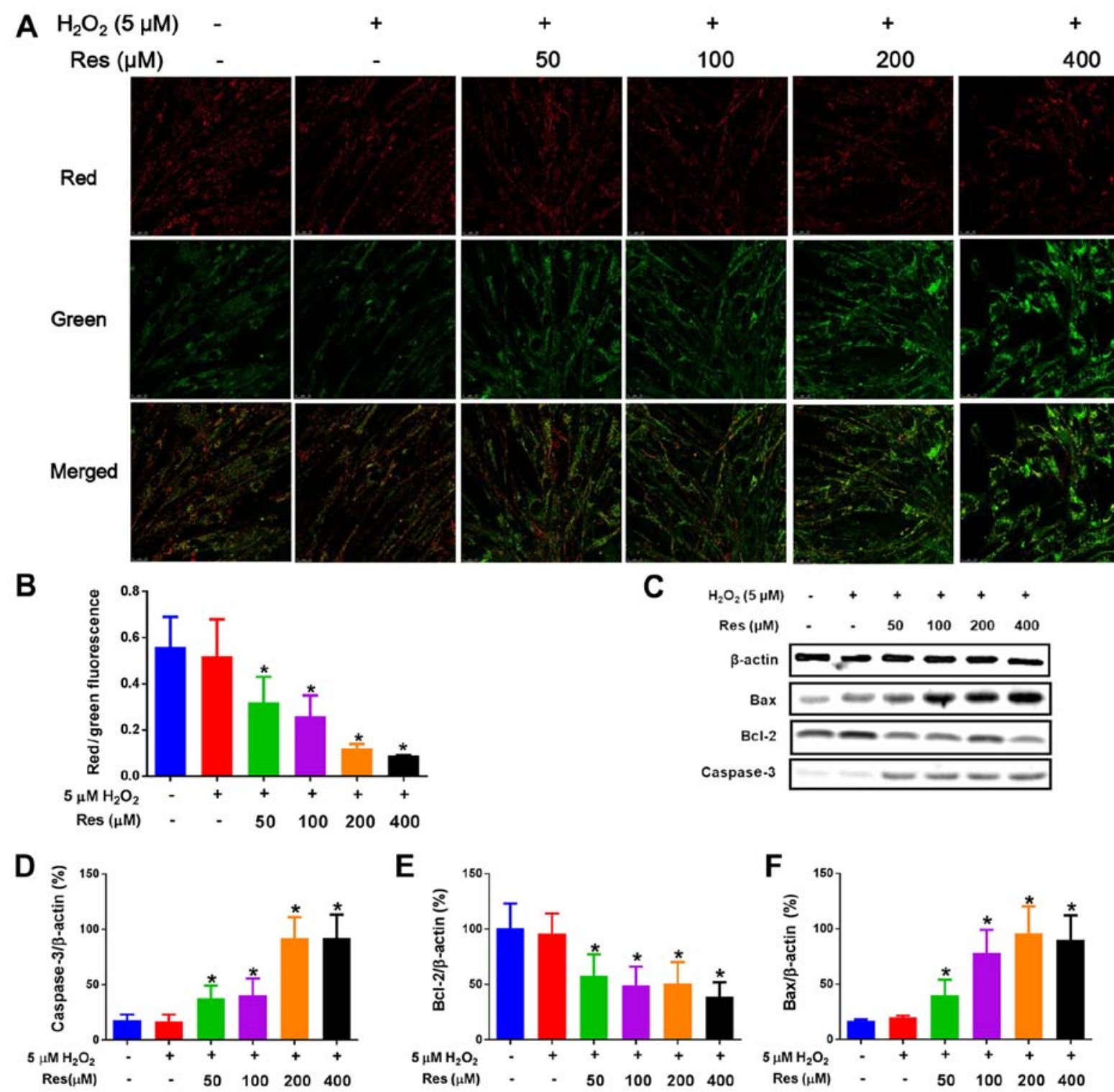

Figure 3. Mitochondrial dysfunction is involved in the process of Res-induced apoptosis in FLSs. (A) $\Delta \psi \mathrm{m}$ was determined using confocal laser scanning microscopy and images were captured (magnification, $\mathrm{x} 400$ ). Red fluorescence represents JC-1 aggregates in matrix of mitochondria, whereas green fluorescence represents JC-1 monomers, indicating a decreased $\Delta \psi \mathrm{m}$. Merged images show the overlap of JC-1 aggregates and monomers. (B) $\Delta \psi \mathrm{m}$ in each group is presented as the red/green fluorescence. In addition, FLSs were lysed and prepared for immunoblotting. (C) Representative images and data showing the expression levels of (D) active caspase-3, (E) Bcl-2 and (F) Bax. Data are shown as the mean \pm standard deviation. ${ }^{*} \mathrm{P}<0.05$, vs. control. $\Delta \psi \mathrm{m}$, mitochondrial membrane potential; FLSs, fibroblast-like synoviocytes; Res, resveratrol; Bcl-2, B-cell lymphoma 2; Bax, Bcl-2-associated X protein.

(Fig. 2A-E). Among the three experimental groups treated with resveratrol, administration of $45 \mathrm{mg} / \mathrm{kg}$ resveratrol exhibited the most marked suppression, which approached the level of normal controls.

Resveratrol induces apoptosis of FLSs within a $5 \mu \mathrm{M}$ $\mathrm{H}_{2} \mathrm{O}_{2}$ environment. Considering the anti-inflammatory and anticancer effects of resveratrol reported previously in the literature, the hypothesis of the present study was that resveratrol triggers the apoptosis of FLSs. In order to test this hypothesis, a TUNEL assay was used to examine the apoptotic rate of the FLSs. As shown in Fig. 2F and G, no evident differences in the apoptotic rates were observed when comparing between the normal controls and FLSs; only when the cells were incubated with $5 \mu \mathrm{M} \mathrm{H}_{2} \mathrm{O}_{2}$ did treatment with resveratrol lead to an evident increase in the apoptotic rate, and this increase occurred in a dose-dependent manner. Subsequently, DCFH-DA was utilized to assess the levels of intracellular ROS, and these results demonstrated that resveratrol led to an increase in the level of intracellular ROS in FLSs in a dose-dependent manner, and therefore it may be potential marker for apoptosis and ER stress (Fig. 2H).

Mitochondrial dysfunction is involved in the process of resveratrol-induced apoptosis in FLSs. As resveratrol induced the apoptosis of FLSs in an environment containing $5 \mu \mathrm{M}$ $\mathrm{H}_{2} \mathrm{O}_{2}$, further elucidation of the intracellular mechanism became the subsequent objective. It has been well established that the mitochondrial signaling pathway has a vital role in cell apoptosis (31). In order to examine whether the mitochondrial signaling pathway is involved in resveratrol-induced apoptosis, the expression levels of mitochondria-associated apoptotic 
A

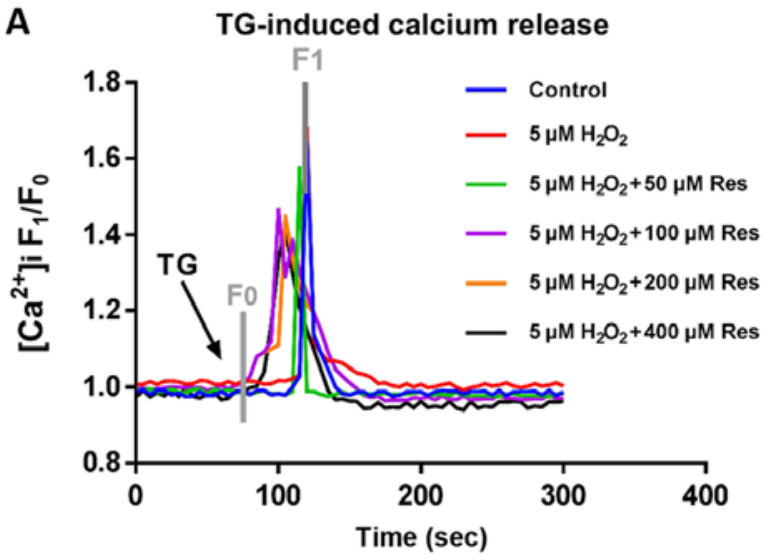

C

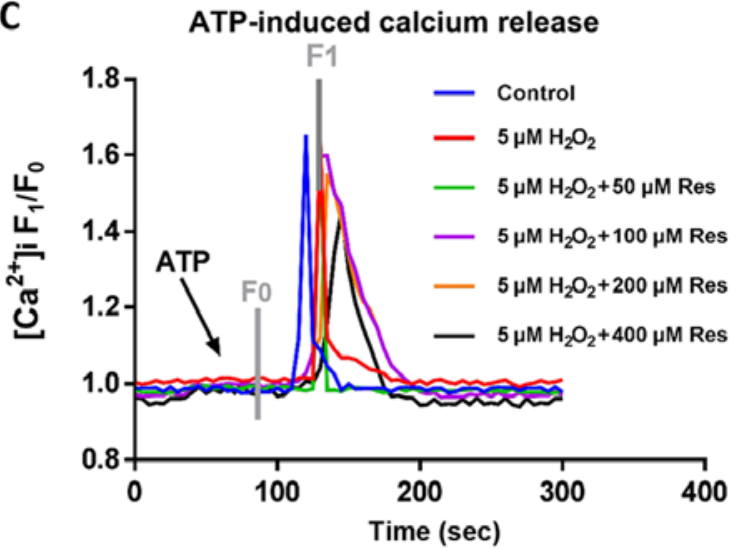

B

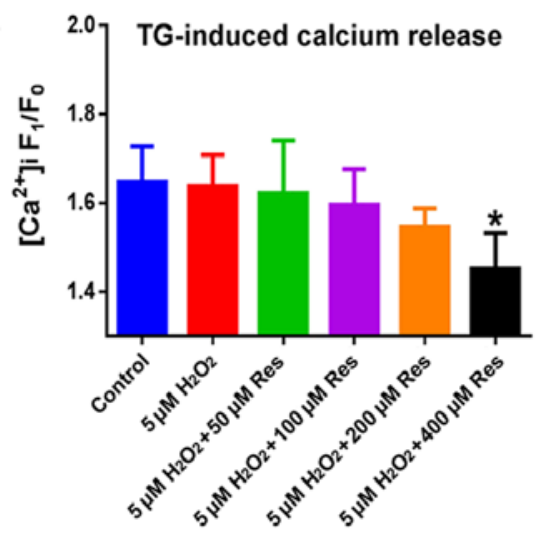

D

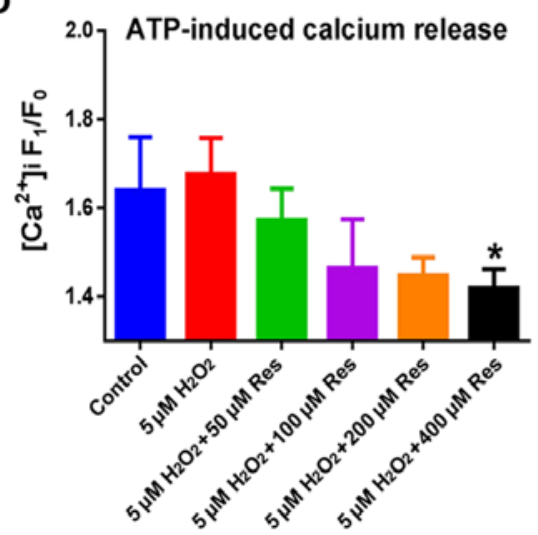

E

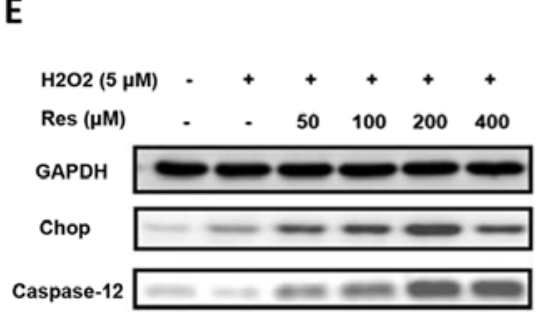

$\mathbf{F}$

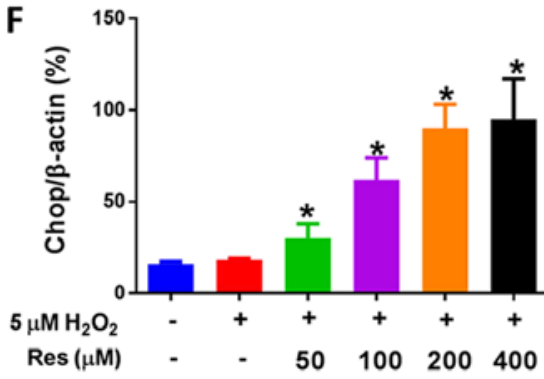

G

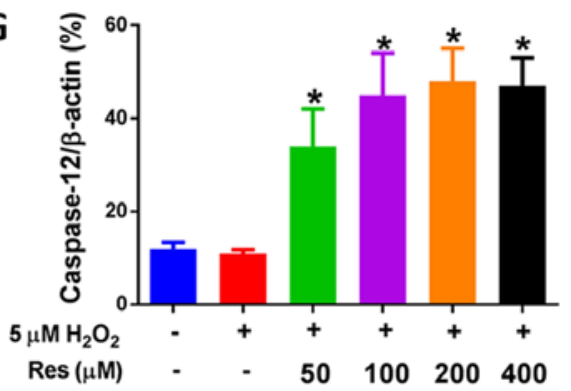

Figure 4. Suppression of $\left[\mathrm{Ca}^{2+}\right]_{\mathrm{i}}$ release and endoplasmic reticulum stress in FLSs are involved in the process of Res-induced apoptosis in FLSs. (A) Representative traces and (B) quantification of data, showing the elevation in $\left[\mathrm{Ca}^{2+}\right]_{\mathrm{i}}$ induced by $2 \mu \mathrm{mol} / 1 \mathrm{TG}$-induced FLSs in Ca ${ }^{2+}$-free saline solution. (C) Representative traces and (D) quantification of data, showing the elevation in $\left[\mathrm{Ca}^{2+}\right]_{\mathrm{i}}$ induced by $100 \mu \mathrm{mol} / 1 \mathrm{ATP}$-induced FLSs in Ca ${ }^{2+}$-free saline solution. Changes in $\left[\mathrm{Ca}^{2+}\right]_{\mathrm{i}}$ were indicated by the ratio of the fluorescence density prior to and following administration of the agonists (F1/F0). (E) Representative images and data showing the expression levels of (F) Chop and $(\mathrm{G})$ caspase-12. Data are shown as the mean \pm standard error of the mean. * $\mathrm{P}<0.05$, vs. control group. Res, resveratrol; $\left[\mathrm{Ca}^{2+}\right]_{\mathrm{i}}$, intracellular calcium concentration; TG, thapsigargin; FLSs, fibroblast-like synoviocytes; Chop, C/EBP-homologous protein.

proteins were initially examined. The immunoblotting results suggested that, in the presence of $5 \mu \mathrm{M} \mathrm{H}_{2} \mathrm{O}_{2}$ treatment, resveratrol led to a notable increase in the expression levels of pro-apoptotic proteins, including Bax and active caspase-3, whereas the anti-apoptotic protein, Bcl-2, was evidently suppressed compared with that in normal control cells and FLSs treated only with $5 \mu \mathrm{M} \mathrm{H}_{2} \mathrm{O}_{2}$ (Fig. 3C-F).

A reduction in $\Delta \psi \mathrm{m}$ or enhanced mitochondrial membrane depolarization have been demonstrated to provide an early sign of cell apoptosis, even in advance of DNA damage (32). JC-1, a widely used fluorescent probe to detect $\Delta \psi \mathrm{m}$, forms aggregates in the mitochondrial matrix that yield red florescence when the value of $\Delta \psi \mathrm{m}$ is relatively high, whereas it forms monomers, giving rise to green florescence, when the value of $\Delta \psi \mathrm{m}$ is comparatively low (33). As shown in Fig. 3A and B, resveratrol was able to suppress the ratio of the red-to-green fluorescent intensity in FLSs in the presence of $5 \mu \mathrm{M} \mathrm{H}_{2} \mathrm{O}_{2}$ in a dose-dependent manner compared with normal control cells and FLSs treated only with $5 \mu \mathrm{M} \mathrm{H}_{2} \mathrm{O}_{2}$, indicating that resveratrol was able to lead to a reduction in $\Delta \psi \mathrm{m}$, which further confirmed the pro-apoptotic effects of resveratrol.

Suppression of $\left[\mathrm{Ca}^{2+}\right]_{i}$ release and ER stress in FLSs are involved in the resveratrol-induced apoptosis of FLSs. Apart from the mitochondria-associated apoptotic pathway, the ER, which is known as an intracellular $\mathrm{Ca}^{2+}$ store, also serves a 


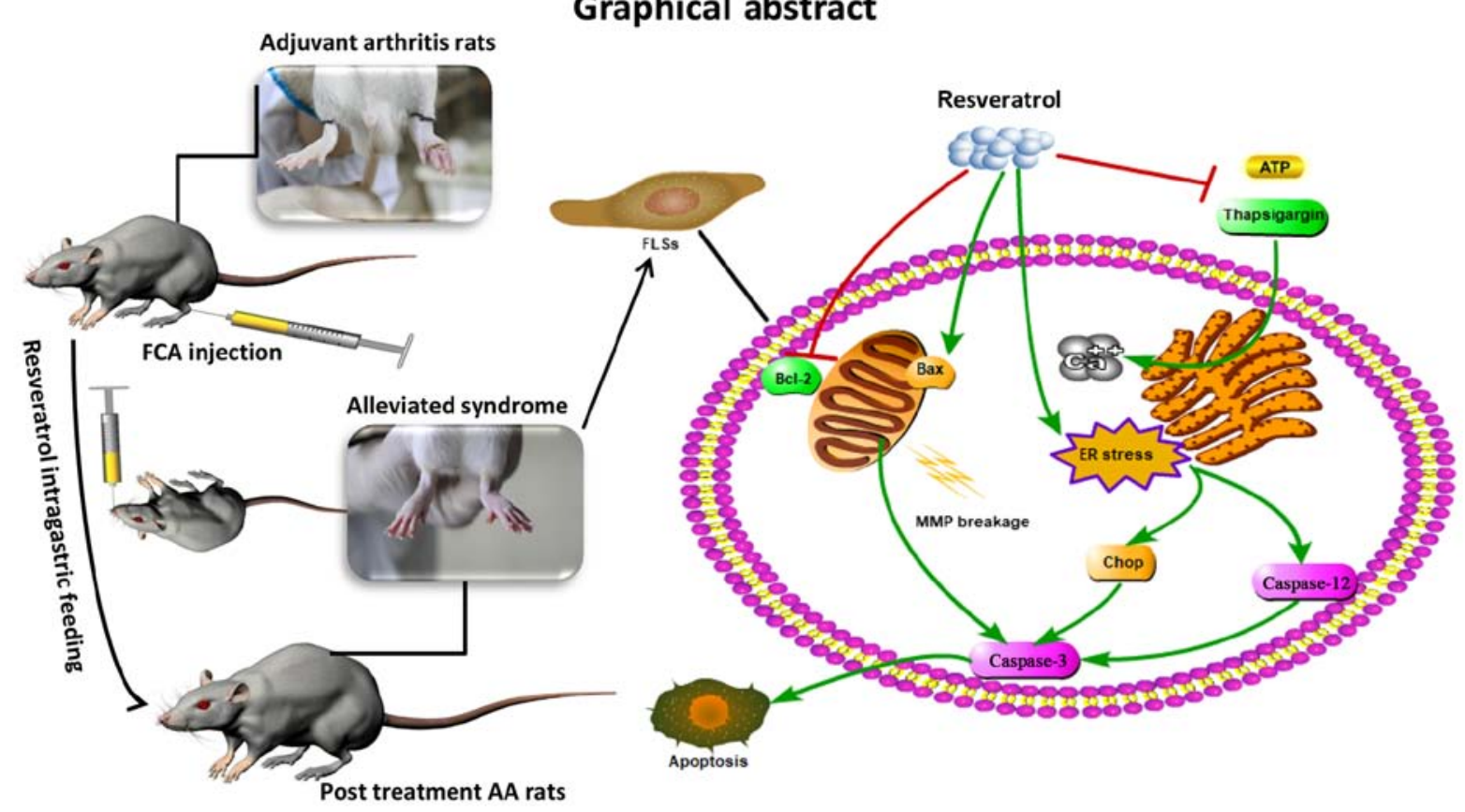

Figure 5. Graphic abstract, illustrating how resveratrol alleviates inflammatory injury and enhances the apoptosis of FLSs via mitochondrial dysfunction and ER stress in AA rats. AA, adjuvant arthritis; FCA, Freund's complete adjuvant; FLSs, fibroblast-like synoviocytes; Bcl-2, B-cell lymphoma 2; Bax, Bcl-2-associated X protein; ER, endoplasmic reticulum; Chop, C/EBP-homologous protein; MMP, matrix metalloproteinase.

crucial role in cell apoptosis. Previous reports have indicated that the depletion of ER $\mathrm{Ca}^{2+}$ stores may trigger cell apoptosis and growth arrest $(34,35)$, and the hypothesis of the present study was that resveratrol may affect the depletion of the $\mathrm{Ca}^{2+}$ store. As $\mathrm{Ca}^{2+}$ stores may be depleted by both ATP and TG, the effect of resveratrol on TG- and ATP-induced $\mathrm{Ca}^{2+}$ release from the ER $\mathrm{Ca}^{2+}$ stores was subsequently examined $(36,37)$. Unexpectedly, the calcium imaging experiments revealed that higher doses of resveratrol led to a suppression of TGand ATP-induced $\mathrm{Ca}^{2+}$ release in FLSs in an environment containing $5 \mu \mathrm{M} \mathrm{H}_{2} \mathrm{O}_{2}$ (Fig. 4A-D). By contrast, continuous and heavily applied ER stress has been suggested to be another possible means of inducing cell apoptosis (38). Two essential terminal apoptosis-associated proteins located downstream of the ER stress pathway, Chop and caspase-12, have been revealed to be elevated under aggravated ER stress (39). In the present study, the immunoblotting data obtained revealed that resveratrol led to an increase in the expression levels of Chop and caspase-12 in FLSs in the presence of $5 \mu \mathrm{M}$ $\mathrm{H}_{2} \mathrm{O}_{2}$, in a dose-dependent manner, compared with levels in normal control cells and FLSs treated only with $5 \mu \mathrm{M}$ $\mathrm{H}_{2} \mathrm{O}_{2}$ (Fig. 4E-G).

\section{Discussion}

In the present study, AA model rats were utilized to examine the features of RA. Resveratrol injection led to an evident reduction in the levels of IL-1, IL-6, IL-8 and TNF- $\alpha$, and an increase in the level of IL-10 in AA rats compared with levels in non-immunized controls in a dose-dependent manner, indicating that resveratrol was able to reduce the level of inflammatory injury, and to enhance the anti-inflammatory capability, in AA rats. RA is an autoimmune disease of the connective tissue characterized by disrupted serum levels of inflammatory cytokines, including IL-1, IL-6, IL-8 and TNF- $\alpha$, which occur during its pathogenesis. The results of the present study further corroborate the therapeutic effects of resveratrol on RA identified in previous studies of others (4-6). Cytokines are vital agents in the inflammatory process. The markedly pro-inflammatory cytokines, IL-1, IL-6, IL-8 and TNF- $\alpha$, together with the markedly anti-inflammatory cytokine, IL-10, were selected for assessment of the properties of resveratrol and its influence on immune responses. Resveratrol is a multifunctional compound, which reduced the serum levels of IL-1, IL-6, IL-8 and TNF- $\alpha$ and simultaneously increased the level of IL-10. It is an important marker in the investigation to identify an effective pharmacological treatment of RA based on the inflammatory etiology. Furthermore, the intragastric administration of resveratrol potently reduced paw swelling, the arthritis score, inflammatory cell infiltration and synovial hyperplasia in AA rats compared with the AA model group (Fig. 1D). Accumulating evidence that resveratrol possesses certain tumor-like properties has previously been reported, based on the fact that it not only suppresses the proliferation of, but also induces apoptosis in various cancer cell types and FLSs (22). Based on our former findings, the proliferation of FLSs was identified as a typical abnormality in RA, and $5 \mu \mathrm{M}$ $\mathrm{H}_{2} \mathrm{O}_{2}$ was identified as the optimal concentration for treatment; this concentration achieved the highest proliferative rate of FLSs compared with higher or lower doses of $\mathrm{H}_{2} \mathrm{O}_{2}$, and simulated the physiological conditions of oxidative stress in AA model rats (22).

The results of the in vitro TUNEL assay revealed that the administration of resveratrol triggered the apoptosis of FLSs in the presence of $5 \mu \mathrm{M} \mathrm{H}_{2} \mathrm{O}_{2}$, and the apoptotic rate was increased with increasing doses of resveratrol. As 
the majority of ROS are produced in mitochondria, and resveratrol was able to reduce excessive ROS production, it was hypothesized that the mitochondrial apoptotic pathway may be involved in the resveratrol-induced apoptosis of FLSs treated with $5 \mu \mathrm{M} \mathrm{H}_{2} \mathrm{O}_{2}$. The levels of pro-apoptotic members of the Bcl family, including Bax and caspase- 3 , were increased, whereas that of the anti-apoptotic protein, Bcl-2, was suppressed following administration of resveratrol in FLSs treated with $5 \mu \mathrm{M} \mathrm{H}_{2} \mathrm{O}_{2}$. Furthermore, a reduced $\Delta \psi \mathrm{m}$ is known to provide an early indicator for cellular apoptosis, and decreased $\Delta \psi \mathrm{m}$ levels were detected upon treatment of resveratrol with $5 \mu \mathrm{M} \mathrm{H}_{2} \mathrm{O}_{2}$.

Apart from the mitochondrial pathway, ER stress may also be involved in the progression of apoptosis. An increasing number of studies have revealed that ER stress, which refers to a particular type of subcellular pathological state of ER that is characterized by calcium dyshomeostasis, accumulation of unfolded proteins, and ER dysfunction caused by intracellular and extracellular stimuli, including viral infection and glucose deficiency, serves vital roles in the process of cell apoptosis (18). During the process of ER stress, the unfolded protein reaction mediated by chaperone-glucose-regulated protein $78 /$ binding immunoglobulin protein and three stress sensors, namely PKR-like ER kinase, activating transcription factor 6 and inositol-requiring enzyme 1, exert a protective role via reducing the accumulation of unfolded protein, and gradually restoring the normal function of ER (40). However, continual and heavily applied ER stress can activate the downstream apoptotic signaling molecules, Chop and caspase-12, leading to cell apoptosis $(20,41)$. In the present study, the levels of these two essential mediators of ER stress-associated apoptosis, Chop and caspase-12, were elevated following treatment with resveratrol in the presence of $5 \mu \mathrm{M} \mathrm{H}_{2} \mathrm{O}_{2}$. As the most important calcium store, ER undertakes vital tasks in maintaining the normal biological activity of the cells, and calcium overload is often linked to ER-associated apoptosis. However, the processes of TG- and ATP-induced calcium release were marginally suppressed following the administration of resveratrol with $5 \mu \mathrm{M} \mathrm{H}_{2} \mathrm{O}_{2}$. This may due to a reduction in the activity of ion channels that mediate the outflow of calcium ions elicited by resveratrol; it is noteworthy that these results were inconsistent with a former study, in which ATP-induced apoptosis was accompanied by an elevated rate of calcium release in HN4 cells (42). In the present study, TG and ATP were used to induce calcium depletion of ER via diverse mechanisms. TG prevents intracellular calcium from flowing into the ER by blocking the sarco/endoplasmic reticulum $\mathrm{Ca}^{2+}$-ATPase (SERCA), leading to a one-way efflux of calcium from the ER to the cytosol. ATP binds to the purinergic receptor (P2Y or $\mathrm{P} 2 \mathrm{X}$ ) on the cell membrane, and subsequently couples with phospholipase $\mathrm{C}$ (PLC), leading to activation of the inositol 1,4,5-trisphosphate receptor on the ER, which subsequently leads to an emptying of the calcium pool (24). Therefore, the suppressive effects of calcium release mediated by resveratrol in FLSs may be associated with an activation of SERCA and inhibition of the P2Y/PLC/IP3 pathway, although this hypothesis requires further confirmation.

In conclusion, the present study demonstrated that resveratrol was able to suppress the level of inflammatory injury in AA and to trigger the apoptosis of FLSs through the mitochondrial pathway and ER stress in the presence of $5 \mu \mathrm{M} \mathrm{H}_{2} \mathrm{O}_{2}$, thereby alleviating the symptoms of AA in SD rats (Fig. 5). However, the effects of resveratrol on the bioactivity of ER-located calcium channels require further investigation, and whether or not other forms of programmed cell death may be involved in this progress remains to be elucidated.

\section{Acknowledgements}

The authors would like to thank Professor Bing Shen (Department of Physiology of Anhui Medical University), who instructed on aspects of this study and who provided the $\left[\mathrm{Ca}^{2+}\right]$ measurements.

\section{Funding}

This study was supported by a grant from the National Natural Science Foundation of China (grant nos. NSFC:81373421 and 81270650).

\section{Availability of data and materials}

The datasets used and/or analyzed during the current study are available from the corresponding author on reasonable request.

\section{Authors' contributions}

JSL, SYF and XYC designed the experiments. JSL and YSZ performed the experiments. JSL and JZY conducted the data analysis. JQZ and WC contributed to data acquisition and analysis. JSL drafted the manuscript.

\section{Ethics approval and consent to participate}

The animal experiments was approved by the Medical Ethics Committee of the Academic Committee at Anhui Medical University. No human experiments were included in the present study.

\section{Patient consent for publication}

Not applicable.

\section{Competing interests}

The authors declare that they have no competing interests.

\section{References}

1. Bellucci E, Terenzi R, La Paglia GM, Gentileschi S, Tripoli A, Tani C and Alunno A: One year in review 2016: Pathogenesis of rheumatoid arthritis. Clin Exp Rheumatol 34: 793-801, 2016.

2. Suzuki A and Yamamoto K: From genetics to functional insights into rheumatoid arthritis. Clin Exp Rheuma 33 (4 Suppl 92): S40-S43, 2015.

3. Shin GC, Kim C, Lee JM, Cho WS, Lee SG, Jeong M, Cho J and Lee K: Apigenin-induced apoptosis is mediated by reactive oxygen species and activation of ERK1/2 in rheumatoid fibroblast-like synoviocytes. Chem Biol Interact 182: 29-36, 2009.

4. Park JS, Kim NR, Lim MA, Kim SM, Hwang SH, Jung KA, Choi J, Park SH and Cho ML: Deficiency of IL-1 receptor antagonist suppresses IL-10-producing B cells in autoimmune arthritis in an IL-17/Th17-dependent manner. Immunol Lett 199: 44-52, 2018. 
5. Jain S, Tran TH and Amiji M: Macrophage repolarization with targeted alginate nanoparticles containing IL-10 plasmid DNA for the treatment of experimental arthritis. Biomaterials 61: $162-177,2015$

6. Khandpur R, Carmona-Rivera C, Vivekanandan-Giri A, Gizinski A, Yalavarthi S, Knight JS, Friday S, Li S, Patel RM, Subramanian V, et al: NETs are a source of citrullinated autoantigens and stimulate inflammatory responses in rheumatoid arthritis. Sci Transl Med 5: 178ra40, 2013.

7. Jackson JK, Higo T, Hunter WL and Burt HM: Topoisomerase inhibitors as anti-arthritic agents. Inflamm Res 57: 126-134, 2008.

8. Bottini N and Firestein GS: Duality of fibroblast-like synoviocytes in RA: Passive responders and imprinted aggressors. Nat Rev Rheumatol 9: 24-33, 2013.

9. DordunooSK, Jackson JK, Arsenault LA, Oktaba AM, Hunter WL and Burt HM: Taxol encapsulation in poly(epsilon-caprolactone microspheres. Cancer Chemother Pharmacol 36: 279-282, 1995.

10. Jackson JK, Tudan C, Sahl B, Pelech SL and Burt HM: Calcium pyrophosphate dihydrate crystals activate MAP kinase in human neutrophils: Inhibition of MAP kinase, oxidase activation and degranulation responses of neutrophils by taxol. Immunology 90 : 502-510, 1997.

11. Hui A, Kulkarni GV, Hunter WL, McCulloch CA and Cruz TF Paclitaxel selectively induces mitotic arrest and apoptosis in proliferating bovine synoviocytes. Arthritis Rheum 40: 1073-1084, 1997.

12. Tian J, Chen JW, Gao JS, Li L and Xie X: Resveratrol inhibits TNF- $\alpha$-induced IL-1 $\beta$, MMP-3 production in human rheumatoid arthritis fibroblast-like synoviocytes via modulation of PI3kinase/Akt pathway. Rheumatol Int 33: 1829-1835, 2013.

13. Quiñonez-Flores CM, González-Chávez SA, Del Río Nájera D and Pacheco-Tena $\mathrm{C}$ : Oxidative stress relevance in the pathogenesis of the rheumatoid arthritis: A systematic review. Biomed Res Int 2016: 6097417, 2016

14. Han G, Xia J, Gao J, Inagaki Y, Tang W and Kokudo N: Anti-tumor effects and cellular mechanisms of resveratrol. Drug Discov Ther 9: 1-12, 2015.

15. Sakamoto T, Horiguchi H, Oguma E and Kayama F: Effects of diverse dietary phytoestrogens on cell growth, cell cycle and apoptosis in estrogen-receptor-positive breast cancer cells. J Nutr Biochem 21: 856-864, 2010.

16. Alkhalaf M: Resveratrol-induced apoptosis is associated with activation of p53 and inhibition of protein translation in T47D human breast cancer cells. Pharmacology 80: 134-143, 2007.

17. Luo H, Yang A, Schulte BA, Wargovich MJ and Wang GY: Resveratrol induces premature senescence in lung cancer cells via ROS-mediated DNA damage. PLoS One 8: e60065, 2013.

18. Faitova J, Krekac D, Hrstka R and Vojtesek B: Endoplasmic reticulum stress and apoptosis. Cell Mol Biol Lett 11: 488-505, 2006.

19. Szegezdi E, Logue SE, Gorman AM and Samali A: Mediators of endoplasmic reticulum stress-induced apoptosis. EMBO Rep 7: 880-885, 2006

20. Mihailidou C, Papazian I, Papavassiliou AG and Kiaris H: CHOP-dependent regulation of p21/waf1 during ER stress. Cell Physiol Biochem 25: 761-766, 2010.

21. Bogeski I, Kilch T and Niemeyer BA: ROS and SOCE: Recent advances and controversies in the regulation of STIM and Orai. J Physiol 590: 4193-4200, 2012.

22. Zhang J, Song X, Cao W, Lu J, Wang X, Wang G, Wang Z and Chen X: Autophagy and mitochondrial dysfunction in adjuvant-arthritis rats treatment with resveratrol. Sci Rep 6: 32928, 2016.

23. Chen X, Lu J, An M, MaZ,Zong H and Yang J: Anti-inflammatory effect of resveratrol on adjuvant arthritis rats with abnormal immunological function via the reduction of cyclooxygenase-2 and prostaglandin E2. Mol Med Rep 9: 2592-2598, 2014.

24. Omatsu-Kanbe M, Inoue K, Fujii Y, Yamamoto T, Isono T, Fujita N and Matsuura H: Effect of ATP on preadipocyte migration and adipocyte differentiation by activating P2Y receptors in 3T3-L1 cells. Biochem J 393: 171-180, 2006.

25. Shu S, Li CM, You YL, Qian XL, Zhou S and Ling CQ Electroacupuncture ameliorates cerebral ischemia-reperfusion injury by regulation of autophagy and apoptosis. Evidence-Based Complement Altern Med 2016: 7297425, 2016.
26. Shen B, Zhu J, Zhang J, Jiang F, Wang Z, Zhang Y, Li J, Huang D, $\mathrm{Ke} D, M a \mathrm{R}$ and Du J: Attenuated mesangial cell proliferation related to store-operated $\mathrm{Ca} 2+$ entry in aged rat: The role of STIM 1 and Orai 1. Age (Dordr) 35: 2193-2202, 2013.

27. Oliver SJ, Firestein GS, Arsenault L, Cruz TF, Cheng TP, Banquerigo ML, Boyle DL and Brahn E: Vanadate, an inhibitor of stromelysin and collagenase expression, suppresses collagen induced arthritis. J Rheumatol 34: 1802-1809, 2007.

28. Kim HR, Kim KW, Kim BM, Lee KA and Lee SH: $\mathrm{N}$-acetyl-1-cysteine controls osteoclastogenesis through regulating Th17 differentiation and RANKL in rheumatoid arthritis. Korean J Intern Med 34: 210-219, 2019.

29. Batooei M, Tahamoli-Roudsari A, Basiri Z, Yasrebifar F, Shahdoust M, Eshraghi A, Mehrpooya M and Ataei S:Evaluating the effect of oral $\mathrm{N}$-acetylcysteine as an adjuvant treatment on clinical outcomes of patients with rheumatoid arthritis: A randomized, double blind clinical trial. Rev Recent Clin Trials 13: 132-138, 2018

30. Paul M, Hemshekhar M, Thushara RM, Sundaram MS NaveenKumar SK, Naveen S, Devaraja S, Somyajit K, West R, Basappa, et al: Methotrexate promotes platelet apoptosis via JNK-mediated mitochondrial damage: Alleviation by $\mathrm{N}$-acetylcysteine and $\mathrm{N}$-acetylcysteine amide. PLoS One 10: e0127558, 2015.

31. Zhang W, Wang X and Chen T: Resveratrol induces mitochondriamediated AIF and to a lesser extent caspase-9-dependent apoptosis in human lung adenocarcinoma ASTC-a-1 cells. Mol Cell Biochem 354: 29-37, 2011.

32. Valdecantos MP, Pérez-Matute P, Quintero P and Martínez JA: Vitamin C, resveratrol and lipoic acid actions on isolated rat liver mitochondria: All antioxidants but different. Redox Rep 15: 207-216, 2010.

33. de la Lastra CA and Villegas I: Resveratrol as an antioxidant and pro-oxidant agent: Mechanisms and clinical implications. Biochem Soc Trans 35: 1156-1160, 2007.

34. Hamada R, Kaminaga K, Suzuki K and Yokoya A: Mitochondrial membrane potential, morphology and atp production in mammalian cells exposed to X-rays. Radiat Prot Dosimetry: Dec 13, 2018. doi: $10.1093 / \mathrm{rpd} / \mathrm{ncy} 254$

35. Sadi G, Bozan D and Yildiz HB: Redox regulation of antioxidant enzymes: Post-translational modulation of catalase and glutathione peroxidase activity by resveratrol in diabetic rat liver. Mol Cell Biochem 393: 111-122, 2014.

36. Walia V, Kakar S and Elble R: Micromanagement of the mitochondrial apoptotic pathway by p53. Front Biosci (Landmark Ed) 16: 749-758, 2011.

37. Bernardi P and Rasola A: Calcium and cell death: The mitochondrial connection. Subcell Biochem 45: 481-506, 2007.

38. Xu Z, Lin S, Wu W, Tan H, Wang Z, Cheng C, Lu L and Zhang X: Ghrelin prevents doxorubicin-induced cardiotoxicity through TNF-alpha/NF-kappaB pathways and mitochondrial protective mechanism. Toxicology 247: 133-138, 2008.

39. Contreras L, Drago I, Zampese E and Pozzan T: Mitochondria: The calcium connection. Biochim Biophys Acta1 797: 607-618, 2010.

40. Fels DR and Koumenis C: The PERK/eIF2alpha/ATF4 module of the UPR in hypoxia resistance and tumor growth. Cancer Biol Ther 5: 723-728, 2006.

41. Zhong J, Kong X, Zhang H, Yu C, Xu Y, Kang J, Yu H, Yi H, Yang X and Sun L: IInhibition of CLIC4 enhances autophagy and triggers mitochondrial and ER stress-induced apoptosis in human glioma U251 cells under starvation. PLoS One 7: e39378, 2012.

42. Xue H, Lu J, Yuan R, Liu J, Liu Y, Wu J, Wu K, Du J and Shen B: Knockdown of CLIC4 enhances ATP-induced HN4 cell apoptosis through mitochondrial and endoplasmic reticulum pathways. Cell Bio 6: 5, 2016.

This work is licensed under a Creative Commons Attribution-NonCommercial-NoDerivatives 4.0 International (CC BY-NC-ND 4.0) License. 